\title{
A cluster-based selective cooperative spectrum sensing scheme in cognitive radio
}

\author{
Nhan Nguyen-Thanh ${ }^{1}$ and Insoo Koo ${ }^{2^{*}}$
}

\begin{abstract}
Developing an effective cooperative spectrum sensing (CSS) scheme in cognitive radio (CR), which is considered as promising system for enhancing spectrum utilization, is necessary. In this paper, a cluster-based optimal selective CSS scheme is proposed for reducing reporting time and bandwidth while maintaining a certain level of sensing performance. Clusters are organized based on the identification of primary signal signal-to-noise ratio value, and the cluster head in each cluster is dynamically chosen according to the sensing data qualities of CR users. The cluster sensing decision is made based on an optimal threshold for selective CSS which minimizes the probability of sensing error. A parallel reporting mechanism based on frequency division is proposed to considerably reduce the time for reporting decision to fusion center of clusters. In the fusion center, the optimal Chair-Vashney rule is utilized to obtain a high sensing performance based on the available cluster's information.
\end{abstract}

Keywords: Cognitive radio, Cooperative spectrum sensing, Cluster, Selective combination, Parallel reporting mechanism

\section{Introduction}

Cognitive radio $(\mathrm{CR})$ has been recently proposed as a promising technology to improve spectrum utilization by enabling secondary access to unused licensed bands. A prerequisite to this secondary access is having no interference to the primary system. This requirement makes spectrum sensing a key function in cognitive radio systems. Among common spectrum sensing techniques, energy detection is an engaging method due to its simplicity and efficiency. However, the major disadvantage of energy detection is the hidden node problem, in which the sensing node cannot distinguish between an idle and a deeply faded or shadowed band [1]. Cooperative spectrum sensing (CSS) which uses a distributed detection model has been considered to overcome that problem [2-12].

Cooperation among CR users (CUs) is usually coordinated by a fusion center (FC). For each sensing interval, CUs will send their sensing data to the FC. In the FC, all local sensing data will be combined to make a final decision on whether the primary signal is present or absent. An optimal data fusion rule was firstly considered

*Correspondence: iskoo@ulsan.ac.kr

2 University of Ulsan, Ulsan, South Korea

Full list of author information is available at the end of the article by Chair and Varshney in [13]. Despite a good performance, the requirement for knowledge of detection and false alarm probabilities at each local node is still a barrier to the optimal fusion rule.

CSS schemes require a large communication resource including sensing time delay, control channel overhead, and consumption energy for reporting sensing data to the FC, especially when the network size is large. There are some previous works [3-9] that considered this problem. In our previous work [3], we proposed an ordered sequential reporting mechanism based on sensing data quality to reduce communication resources. A similar sequential ordered report transmission approach was considered for reducing the reporting time in [4]. However, the reporting time of these methods is still unpredictably long. In [5], the authors proposed to use a censored truncated sequential spectrum sensing technique for saving energy. On the other hand, cluster-based CSS schemes are considered for reducing the energy of CSS [6] and for minimizing the bandwidth requirements by reducing the number of terminals reporting to the fusion center [7]. In [8], Chen et al. proposed a cluster-based CSS scheme to optimize the cooperation overhead along with the sensing reliability. In fact, these proposed cluster schemes can reduce the amount of direct cooperation with the FC but cannot 
reduce the communication overhead between CUs and the cluster header. A similar problem can be observed in the cluster scheme in [9], though the optimal cluster size to maximize the throughput used for negotiation is identified. Another consideration of the cluster scheme is to enhance sensing performance when the reporting channel suffers from a severe fading environment $[10,11]$.

In this paper, we propose a cluster-based selective CSS scheme which utilizes an efficient selective method for the best quality sensing data and a parallel reporting mechanism. The selective method, which is usually adopted in cooperative communications [14,15], is applied in each cluster to implicitly select the best sensing node during each sensing interval as the cluster header without additional collaboration among CUs. The parallel reporting mechanism based on frequency division is considered to strongly reduce the reporting time of the cluster decision. In the $\mathrm{FC}$, the optimal Chair-Vashney rule (CV rule) is utilized to obtain a high sensing performance based on the available cluster's signal-to-noise ratio (SNR). In this way, the proposed cooperative sensing will be performed with an extremely low cooperation resource while a certain high level of sensing performance is ensured.

The remainder of this paper is organized as follows. In Section 2, some background on spectrum sensing and optimal fusion rule is described. In Section 3, we present system descriptions. The proposed system model and detailed descriptions of the proposed cluster-based selective CSS scheme are also given in Section 4. Simulation results are shown in Section 5. Finally, the conclusions are drawn in Section 6.

\section{Preliminaries}

\subsection{Local spectrum sensing}

Each CU conducts a spectrum sensing process, which is called local spectrum sensing in distributed scenario for detecting the primary user (PU) signal. Local spectrum sensing at the $i$ th $\mathrm{CU}$ is essentially a binary hypotheses testing problem:

$$
\left\{\begin{array}{l}
H_{0}: x_{i}(t)=n_{i}(t) \\
H_{1}: x_{i}(t)=h_{i} s(t)+n_{i}(t)
\end{array}\right.
$$

where $H_{0}$ and $H_{1}$ correspond, respectively, to hypotheses of absence and presence of the PU signal, $x_{i}(t)$ represents received data at $\mathrm{CU}_{i}, h_{i}$ denotes the gain of the channel between the PU and the $\mathrm{CU}_{i}, s(t)$ is the signal transmitted from the primary user, and $n(t)$ is additive white Gaussian noise. Additionally, channels corresponding to different CUs are assumed to be independent, and further, all CUs and PUs share a common spectrum allocation.

Among various methods for spectrum sensing, energy detection has been shown to be quite simple, quick, and able to detect the primary signal - even if the feature of the primary signal is unknown. Here, we consider the energy detection for local spectrum sensing. Figure 1 shows the block diagram of an energy detection scheme. To measure the signal power in a particular frequency region in a time domain, a band-pass filter is applied to the received signal, and power of the signal samples is then measured at $\mathrm{CU}$. The estimation of received signal power is given at $\mathrm{CU}_{i}$ by the following equation:

$$
x_{E_{i}}=\sum_{j=1}^{N}\left|x_{j}\right|^{2},
$$

where $x_{j}$ is the $j$ th sample of the received signal and $N=$ $2 T W$ in which $T$ and $W$ correspond to detection time and signal bandwidth in hertz, respectively.

If the primary signal is absent, $x_{E_{i}}$ follows a central chisquare distribution with $N$ degrees of freedom; otherwise, $x_{E_{i}}$ follows a noncentral chi-square distribution with $N$ degrees of freedom and a noncentrality parameter $\theta_{i}=$ $N \gamma_{i}$, i.e.,

$$
x_{E_{i}} \sim\left\{\begin{array}{cc}
\chi_{N}^{2}, & H_{0} \\
\chi_{N}^{2}\left(\theta_{i}\right) & H_{1}
\end{array}\right.
$$

When $N$ is relatively large (e.g., $N>200$ ) [16], $x_{E}$ can be well approximated as a Gaussian random variable under both hypotheses $H_{1}$ and $H_{0}$, according to the central limit theorem such that

$$
x_{E_{i}} \sim\left\{\begin{array}{cc}
\mathcal{N}(N, 2 N), & H_{0} \\
\mathcal{N}\left(N\left(1+\gamma_{i}\right), 2 N\left(1+2 \gamma_{i}\right)\right) & H_{1}
\end{array}\right.
$$

where $\gamma_{i}$ is the SNR of the primary signal at the CU.

For the case of local sensing or hard decision fusion, the CUs will make the local sensing decision based on an energy threshold $\lambda_{i}$ as follows:

$$
D_{i}=\left\{\begin{array}{cc}
1, & x_{E_{i}}>\lambda_{i} \\
-1, & \text { otherwise }
\end{array}\right.
$$

where $D_{i}=1$ and $D_{i}=-1$ mean that the hypotheses of $H_{1}$ and $H_{0}$ are declared at the $i$ th $\mathrm{CU}$, respectively. The local probability of detection and the local probability of false alarm can be determined based on (4) by:

$$
p_{f_{i}}=\operatorname{Pr}\left[D_{i}=1 \mid H_{0}\right]=Q\left(\frac{\lambda_{i}-N}{\sqrt{2 N}}\right)
$$

and

$$
p_{d_{i}}=\operatorname{Pr}\left[D_{i}=1 \mid H_{1}\right]=Q\left(\frac{\lambda_{i}-N\left(1+\gamma_{i}\right)}{\sqrt{2 N\left(1+2 \gamma_{i}\right)}}\right),
$$

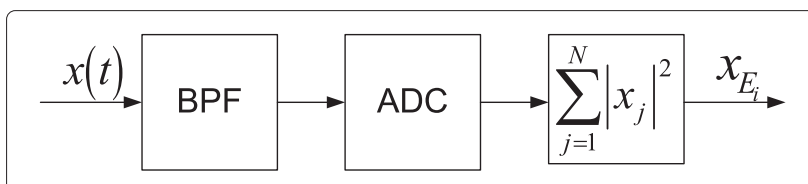

Figure 1 Block diagram of the energy detection scheme. 
respectively, where $Q$ (.) is the Marcum-Q function, i.e., $Q(x)=\frac{1}{\sqrt{2 \pi}} \int_{x}^{\infty} e^{-\frac{t^{2}}{2} d t}$.

\subsection{The optimal fusion rule for global decision}

Chair and Varshney provided the optimal data fusion rule in a distributed local hard decision detection system [13]. This optimal rule is in fact the sum of weighted local decisions where the weights are functions of probabilities of detection and false alarm.

The optimal fusion rule is based on the likelihood ratio test as follows:

$$
\frac{\operatorname{Pr}\left[D_{1}, \ldots, D_{n} \mid H_{1}\right]}{\operatorname{Pr}\left[D_{1}, \ldots, D_{n} \mid H_{0}\right]} \underset{H_{1}}{\gtrless} \frac{\pi_{0}\left(C_{10}-C_{00}\right)}{\pi_{1}\left(C_{01}-C_{11}\right)},
$$

where $\pi_{0}$ and $\pi_{1}$ are the prior probabilities of the presence and absence of the PU signal, respectively, and $C_{00}, C_{01}$, $C_{10}$, and $C_{11}$ are the decision costs. If we choose $C_{00}=$ $C_{11}=0$ and $C_{01}=C_{10}=1$, the likelihood ratio test now follows the minimum probability of error criterion [17], and (8) can be rewritten as:

$$
\frac{\operatorname{Pr}\left[D_{1}, \ldots, D_{n} \mid H_{1}\right]}{\operatorname{Pr}\left[D_{1}, \ldots, D_{n} \mid H_{0}\right]} \underset{H_{1}}{\gtrless} \frac{H_{0}}{\pi_{1}},
$$

Since the decision set $\left\{D_{i}\right\}$ is independent, the loglikelihood ratio test corresponding to (9) is as follows:

$$
\log \frac{\prod_{i=1}^{n} \operatorname{Pr}\left[D_{i} \mid H_{1}\right]}{\prod_{i=1}^{n} \operatorname{Pr}\left[D_{i} \mid H_{0}\right]} \underset{H_{1}}{\gtrless} \log \frac{\pi_{0}}{\pi_{1}},
$$

If $S+$ and $S$-denote the set of all $i$ such that $\left\{D_{i}=1\right\}$ and $\left\{D_{i}=-1\right\}$, respectively, then (10) can be computed by:

$$
\sum_{S+} \frac{\operatorname{Pr}\left[D_{i}=1 \mid H_{1}\right]}{\operatorname{Pr}\left[D_{i}=1 \mid H_{0}\right]}+\sum_{S-} \frac{\operatorname{Pr}\left[D_{i}=-1 \mid H_{1}\right]}{\operatorname{Pr}\left[D_{i}=-1 \mid H_{0}\right]} \underset{H_{1}}{\gtrless} \log \frac{\pi_{0}}{\pi_{1}} .
$$

Finally, the Chair-Vashney fusion rule can be rewritten in the form of the weighting formula as follows:

$$
W_{0}+\sum_{i=1}^{n} W_{i} D_{i} \stackrel{H_{0}}{\gtrless} 0,
$$

where $W_{0}=\log \frac{\pi_{1}}{\pi_{0}}$ and

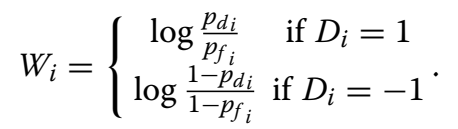

Local false alarm probability $p_{f_{i}}$ and local detection probability $p_{d_{i}}$ are defined in (6) and (7), respectively.

\section{System description}

In this paper, the CR network, which shares the same spectrum band with a license system, utilizes a cluster-based CSS scheme as shown in Figure 2. The CR network is organized in multiple clusters in each of which the CUs have an identical average SNR of the received primary signal. This identical SNR assumption can be practical when the clusters are divided according to geographical position, i.e., adjacent CUs in a small area are gathered into a cluster. The header in each cluster is not fixed but dynamically selected for each sensing interval based on the quality of the sensing data at each CU. In detail, the node with the most reliable sensing result will take on the cluster header's roles which include making and reporting the cluster's decision to the FC. In order to reduce the reporting time and bandwidth, only the sensing data of the cluster header, which is the most reliable sensing data, is utilized to make the cluster decision. This method means that the decision of a cluster is made according to the selective combination method. The FC will combine all cluster decisions to make a final decision and broadcast the final sensing decision to the whole network.

The fusion rule in the FC can be any kind of hard decision fusion rules such as an OR rule, AND rule, 'K out of N' rule, or Chair-Varshney rule. Without loss of generality, we propose the utilization of the optimal Chair-Varshney rule at the FC since the SNR value of the received primary signal at the $\mathrm{CU}$ is available in this proposed scheme. However, there are three issues with the proposed scheme that need to be considered:

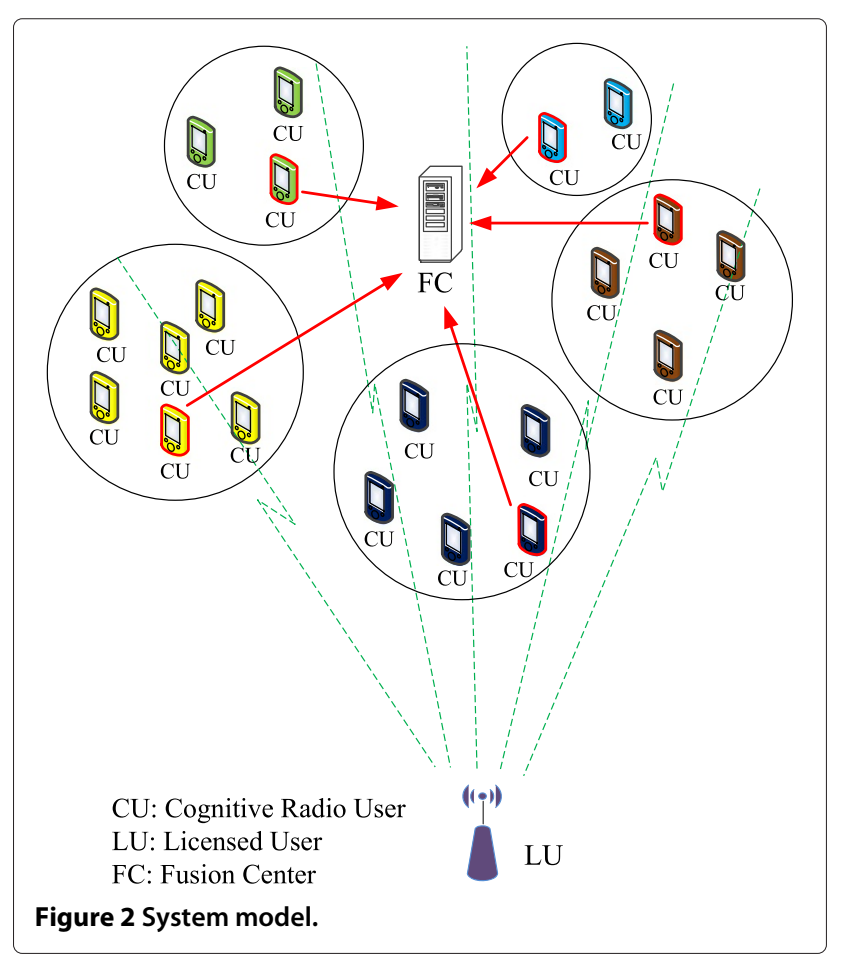


1. How can the scheme efficiently select the cluster header, which is the node with the best quality for sensing data, for each sensing interval without any extra overhead among nodes in the cluster?

2. How can the cluster header optimally make the cluster decision?

3. What is the method for reporting the cluster decision to the FC?

The answers to these questions are given in the following section.

\section{The proposed cluster-based selective CSS scheme}

\subsection{Selective CSS mechanism}

In this subsection, we suggest a cluster header selection based on sensing data reliability. For each sensing interval, the $\mathrm{CU}$ with the most reliable sensing data in a cluster is selected to be the cluster header. Obviously, the reliability of the sensing data can be evaluated by the log-likelihood ratio (LLR) of the sensing result. The LLR value of the received signal energy $x_{E_{i}}$ is given by:

$$
\Lambda_{i}=\log \left(\frac{f_{X \mid H_{1}}\left(x_{E_{i}} \mid H_{1}\right)}{f_{X \mid H_{0}}\left(x_{E_{i}} \mid H_{0}\right)}\right)
$$

where $f_{X \mid H_{j}}\left(x_{E_{i}} \mid H_{j}\right), j=0,1$ is the probability density function (PDF) of $x_{E_{i}}$ corresponding to each hypothesis. Since the SNRs of the received primary signals in a cluster are identical, the LLR of the $i$ th user in the $c_{j}$ th cluster $\Lambda_{i, c_{j}}$ can be considered to be derived from the same distribution $f_{\Lambda_{c_{j}}}(\Lambda)$ of LLR $\Lambda$. For each cluster, therefore, the LLR value can be normalized such that it has a zero mean as follows:

$$
Y_{i, c_{j}}=\Lambda_{i, c_{j}}-E\left[\Lambda_{c_{j}}\right] .
$$

It is obvious that the reliability of the sensing data will be higher if the absolute value of the normalized LLR is larger. We propose utilization of the absolute value of the normalized LLR $\left|Y_{i, c_{j}}\right|$ as the reliability coefficient for selecting the cluster header as well as the selective cluster data.

In order to implicitly select the most reliable sensing data among CUs in a cluster without additional data collaboration, one contention time should be determined for each CU as follows:

$$
t_{\text {con }}=\exp \left(-\kappa\left|Y_{i, c_{j}}\right|\right) \text {, }
$$

where $\kappa$ is a predefined constant such that the contention time is sufficient. Obviously, from this equation, the node with the highest absolute value of the normalized LLR will have the smallest contention time. In contention, each $\mathrm{CU}$ must monitor the reporting channel and wait for a quiescent condition before considering itself as a cluster header, i.e., the node with the most reliable sensing data, when the contention time expires. The $\mathrm{CU}$ who wins the contention will make a local cluster decision and report the cluster decision to the FC based on its own sensing data as follows:

$$
\begin{gathered}
H_{1}: D_{c_{j}}=1 \\
Y_{c_{j}} \stackrel{D^{2}}{\gtrless} \tau_{c_{j}}, \\
H_{0}: D_{c_{j}}=-1
\end{gathered}
$$

where $Y_{c_{j}}$ is equal to the normalized LLR with highest absolute value and $\tau_{c_{j}}$ is the cluster threshold. Next, we consider the problem of choosing the optimal cluster threshold.

\subsection{Cluster threshold determination}

In order to make a controllable cluster decision that follows a certain criterion such as the Neyman-Pearson criterion or minimum error probability criterion, one factor to consider is the probability density function of the cluster's selective sensing data which is utilized to make the cluster decision. In this subsection, we will formulate this requirement.

First, from (15), the normalized LLR distributions of a $\mathrm{CU}$ in the $c_{j}$ th cluster are given by:

$$
\left\{\begin{array}{l}
F_{Y_{c_{j}}}(y)=F_{\Lambda_{c_{j}}}\left(y+E\left[\Lambda_{c_{j}}\right]\right) \\
f_{Y_{c_{j}}}(y)=f_{\Lambda_{c_{j}}}\left(y+E\left[\Lambda_{c_{j}}\right]\right)
\end{array},\right.
$$

where $F_{\Lambda_{c_{j}}}(\Lambda)$ and $f_{\Lambda_{c_{j}}}(\Lambda)$ are the cumulative distribution function (CDF) and PDF of the LLR of the received primary signal power at the CUs in the $c_{j}$ th cluster, respectively. These LLR distributions are given by:

$$
\left\{\begin{array}{l}
F_{\Lambda_{c_{j}}}(\Lambda)=\pi_{0} F_{\Lambda_{c_{j}} \mid H_{0}}\left(\Lambda \mid H_{0}\right)+\pi_{1} F_{\Lambda_{c_{j}} \mid H_{1}}\left(\Lambda \mid H_{1}\right) \\
f_{\Lambda_{c_{j}}}(\Lambda)=\pi_{0} f_{\Lambda_{c_{j}} \mid H_{0}}\left(\Lambda \mid H_{0}\right)+\pi_{1} f_{\Lambda_{c_{j}} \mid H_{1}}\left(\Lambda \mid H_{1}\right)
\end{array}\right.
$$

where the conditional PDF's of the LLR under $H_{0}$ and $H_{1}$ are determined in [12] as follows:

$$
\begin{aligned}
& \text { If } \Lambda \geq-\frac{b}{a} \\
& \qquad\left\{\begin{array}{l}
f_{\Lambda \mid H_{0}}(\Lambda)=\frac{b e^{-\left(\frac{\sqrt{a+b \Lambda}-N / 2}{2 \sqrt{N}}\right)^{2}}}{4 \sqrt{\pi N} \sqrt{a+b \Lambda}} \\
f_{\Lambda \mid H_{1}}(\Lambda)=\frac{b e^{-\left(\frac{\sqrt{a+b \Lambda}-N(2 \gamma+1) / 2}{2 \sqrt{N(2 \gamma+1)}}\right)^{2}}}{4 \sqrt{\pi N(2 \gamma+1)} \sqrt{a+b \Lambda}}
\end{array}\right.
\end{aligned}
$$

and

$$
\left\{\begin{array}{l}
F_{\Lambda \mid H_{0}}(\Lambda)=1-\frac{1}{2} \operatorname{erfc}\left(\frac{\sqrt{a+b \Lambda}-N / 2}{2 \sqrt{N}}\right) \\
F_{\Lambda \mid H_{1}}(\Lambda)=1-\frac{1}{2} \operatorname{erfc}\left(\frac{\sqrt{a+b \Lambda}-N(2 \gamma+1) / 2}{2 \sqrt{N(2 \gamma+1)}}\right)
\end{array}\right.
$$

where $a=\left[N^{2} / 4+N \log (2 \gamma+1) / \gamma\right](2 \gamma+1)$ and $b=2 N(2 \gamma+1) / \gamma$.

Otherwise, $F_{\Lambda \mid H_{0}}(\Lambda)=0, F_{\Lambda \mid H_{1}}(\Lambda)=0$,

$f_{\Lambda \mid H_{0}}(\Lambda)=0$, and $f_{\Lambda \mid H_{1}}(\Lambda)=0$. 
Since the SNRs of the received primary signal at the CUs in a cluster are identical and the selective data for a cluster is the highest absolute value of the normalized LLR, the distribution of the selective cluster data will be equal to the distribution of the $n_{0}$ th absolute order sample, i.e., the sample with the highest absolute value, where $n_{0}$ is the number of CUs in the cluster. In addition, the PDF of the $n_{0}$ th absolute order sample is given by:

$$
f_{Y_{\left(n_{0}\right), c_{j}}}(y)=n_{0}\left|F_{Y_{c_{j}}}(y)-F_{Y_{c_{j}}}(-y)\right|^{n_{0}-1} f_{Y_{c_{j}}}(y) \text {. }
$$

The derivation of (22) can be found in the Appendix. Similarly, the conditional PDF of the $n_{0}$ th absolute order sample under the $H_{j}$ hypothesis, $j=0,1, f_{Y_{\left(n_{0}\right), c_{j}} \mid H_{j}}(y)$ can be achieved.

For a specific value of threshold $\tau_{c_{j}}$, the probability of false alarm and the probability of detection of the $c_{j}$ th cluster are, respectively, given by:

$$
P_{F_{c_{j}}}=\int_{\tau_{c_{j}}}^{+\infty} f_{Y_{\left(n_{0}\right), c_{j}} \mid H_{0}}(y) d y
$$

and

$$
P_{D_{c_{j}}}=\int_{\tau_{c_{j}}}^{+\infty} f_{Y_{\left(n_{0}\right), c_{j}} \mid H_{1}}(y) d y .
$$

Since the probabilities of false alarm and the probability of detection of the $c_{j}$ th cluster in (23) and (24) mainly depend on the received primary signal SNR and the number of nodes in the cluster, the cluster threshold can be determined off-line in the initial phase of the cluster establishment based on the Neyman-Pearson criterion or the minimum error probability criterion. For the Neyman-Pearson criterion, the probability of false alarm $P_{F_{c_{j}}}$ is predefined. Also, the cluster threshold $\tau_{c_{j}}$ is computed based on (23). In this paper, we utilize the minimum error probability criterion to numerically determine the optimal cluster threshold through the following equation:

$$
\tau_{c_{j}}^{\mathrm{opt}}=\underset{\tau_{c_{j}}}{\arg \min }\left[\pi_{0} P_{F_{c_{j}}}+\pi_{1}\left(1-P_{D_{c_{j}}}\right)\right] .
$$

\subsection{Parallel report mechanism}

For implementing the proposed selective mechanism in a cluster, all CUs in a cluster have to monitor the control channel to determine the cluster header during the contention time. One question raised here is how to arrange the contention time for multiple clusters in the network. Generally, there are two common solutions for this problem. The first approach is to assume that the contention times of the clusters are carried out sequentially over time. This method requires a strict synchronization among CUs in the network and a long contention time to minimize the collision in contention due to differences in transmission range. Obviously, this method can cause a long reporting time with a high rate of contention collision. The second approach is to assume that the contention times of different clusters are conducted in parallel with different subcontrol channels. Since each cluster only reports a 1-bit hard decision to the FC, the subcontrol channel can be reduced to a pair of frequencies corresponding to two possible values of a cluster decision. This means that a node in a certain cluster only monitors two predetermined frequencies during the contention time, and the node who wins the contention will transmit only one predefined frequency to the FC according to its cluster decision. Normally, a control channel bandwidth is sufficient for allocating a reasonable number of frequency pairs to clusters. For example, it is acceptable to divide 50 pairs of frequencies for 50 clusters in a $200-\mathrm{kHz}$ control channel. Figure 3 shows an example of a sensing frame structure for the proposed parallel report mechanism compared with the conventional fixed allocation direct reporting method.

In this method, the problems of strict synchronization and contention collision, which can occur with the previous method, are completely resolved. Indeed, with this parallel contention and reporting mechanism, the synchronization among CUs can be looser since there is only one contention time that is identical to the reporting time. No collision between two cluster reports will occur since these cluster decisions are transmitted at different frequencies. Even in the case that two CUs in a cluster have the same value of the most reliable sensing data, a collision still will not occur since the two nodes will transmit the same frequency, and at the receiver side, two transmitted frequencies can be considered as two versions of a multipath signal. The remainder problem with this parallel reporting method is that the FC needs to be equipped with parallel communication devices such as an FFT block, which is usually used in an OFDM receiver, or a filter bank block to detect multiple reporting frequencies. However, this requirement is not a big issue.

\section{Simulation results}

The simulation of the proposed cluster-based selective CSS scheme is conducted under the following assumptions:

- The LU signal is a DTV signal as in [18].

- The bandwidth of the PU signal is $6 \mathrm{MHz}$, and the AWGN channel is considered.

- The local sensing time is $50 \mu \mathrm{s}$.

- The probability of the presence and absence of PU signal is 0.5 for both.

- The network has $N_{0}$ nodes and can be divided into $n_{c}$ clusters. Each cluster includes $n_{0}$ nodes. 


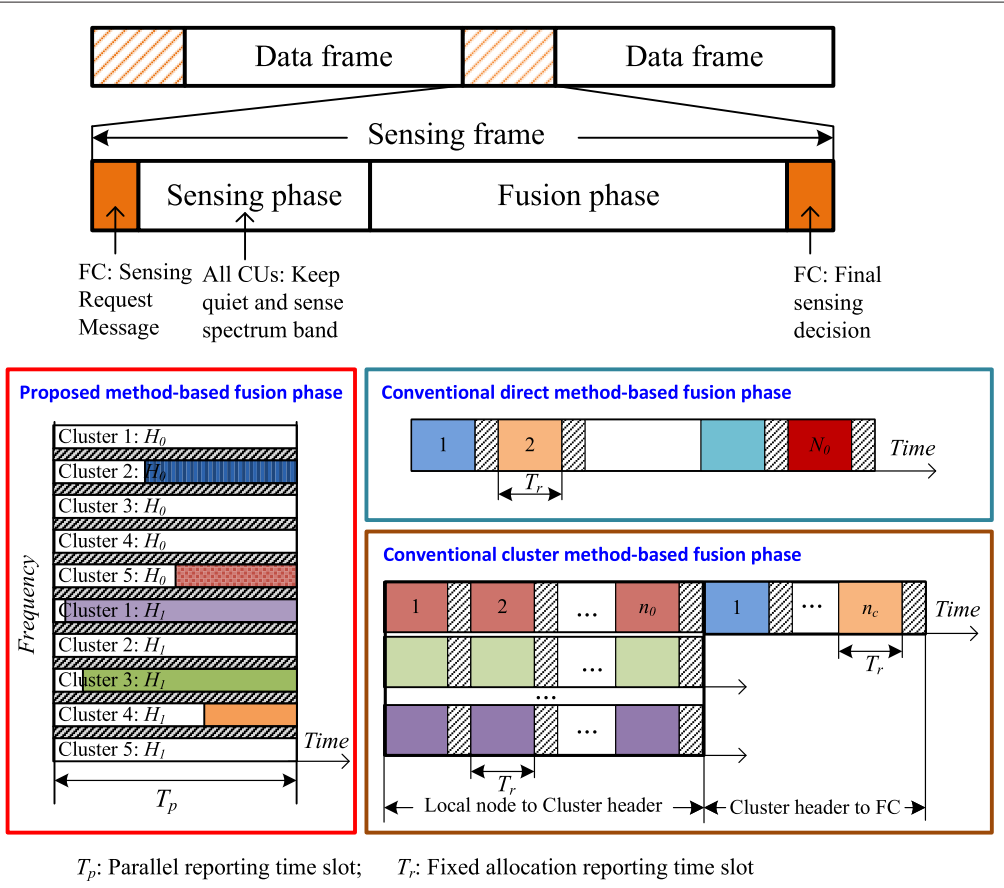

Figure 3 Sensing frame structure.

First, we evaluate the sensing performance of the selective method in the cluster with three different received primary signal SNRs of $-14,-12$, and $-10 \mathrm{~dB}$ when the number of nodes in the cluster changes from 1 to 100 . As shown in Figure 4, the probability of error will decrease along with the increase in the number of nodes in the cluster. However, the decreasing rate of probability of error is low when the number of nodes in the cluster is large,

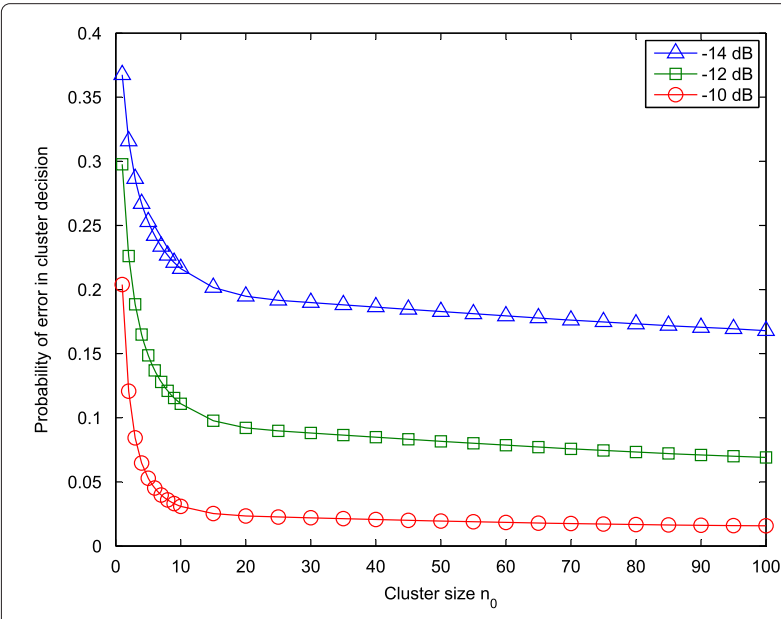

Figure 4 Probability of sensing error in a cluster decision of the proposed selective method. This is for different numbers of nodes in the cluster when the received primary signal SNR is $-14,-12$, and $-10 \mathrm{~dB}$. specially when $n_{0}>10$. Therefore, the selective method only provides high sensing efficiency when the number of nodes is in the range of 20.

Second, we assume that the network includes five clusters with different SNR values corresponding to -20 , $-18,-16,-14$, and $-12 \mathrm{~dB}$. The error probabilities of the global $\mathrm{CV}$ rule-based conventional direct reporting scheme, the cluster and global CV rule-based conventional cluster reporting scheme, and the proposed CSS scheme are then observed according to different values of cluster size. As illustrated in Figure 5, the error probabilities of all CSS schemes decrease along with the increase of the cluster size. The direct conventional CV rulebased CSS scheme provides the best sensing performance. The proposed CSS scheme outperforms the cluster and global CV rule-based conventional cluster CSS scheme when the cluster size is small, i.e., $n_{0}<8$. When the cluster size is large, i.e., $n_{0}>8$, the sensing error probability of the proposed method is slightly higher than that of the conventional cluster scheme, which utilizes a CV rule at both cluster headers and FC. However, it is noteworthy that the cost of this better performance with the conventional cluster and direct schemes compared with the proposed scheme are the extremely large amount of overhead, energy consumption, and reporting time for collecting all decisions from all nodes in the network.

Finally, to clarify the energy efficiency and collection time savings, we first assume that $E_{0}=k E_{1}$ where $E_{0}$ 


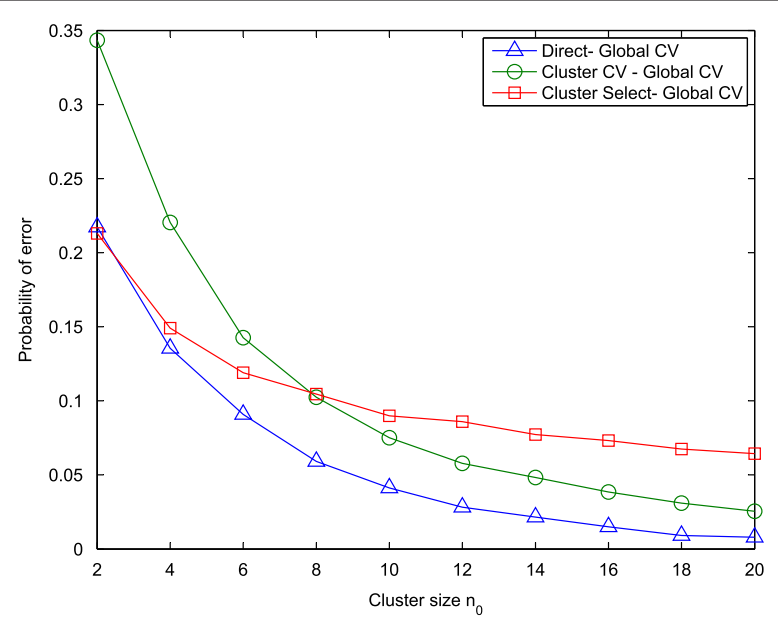

Figure 5 Probability of sensing error of the proposed and conventional CSS schemes. Probability of sensing error of the direct conventional CV rule-based scheme, the cluster and global CV rule-based conventional cluster reporting scheme, and the proposed CSS schemes for different cluster sizes when the network includes five clusters with a SNR value corresponding to $-20,-18,-16,-14$, and $-12 \mathrm{~dB}$, respectively.

is the energy for transmitting the report from a cluster header to the FC and $E_{1}$ is the energy for transmitting the report from a local node to the cluster header. Similarly, we assume that $T_{p}=l T_{r}$ where $T_{p}$ is the parallel reporting time slot, and $T_{r}$ is the fixed allocation reporting time slot (see Figure 3). We also assume that each cluster utilizes a separate reporting channel to transmit the sensing result from local nodes to the cluster header in the case of a conventional cluster-based CSS scheme, and the guard interval between time slots is ignored. As a result, the reporting energy consumption and the total reporting time of the direct reporting (DIR), the conventional cluster (CON), and the proposed (PROP) CSS schemes can be calculated by:

$$
\left\{\begin{array}{l}
E_{\mathrm{DIR}}=N_{0} E_{0}=n_{c} n_{0} E_{0} \\
E_{\mathrm{CON}}=n_{c} E_{0}+\left(n_{0}-1\right) n_{c} E_{1} \\
E_{\mathrm{PROP}}=n_{c} E_{0}
\end{array}\right.
$$

and

$$
\left\{\begin{array}{l}
T_{\mathrm{DIR}}=N_{0} T_{r}=n_{c} n_{0} T_{r} \\
T_{\mathrm{CON}}=n_{c} T_{r}+n_{0} T_{r}=\left(n_{c}+n_{0}\right) T_{r} \\
T_{\mathrm{PROP}}=T_{p}=l T_{r}
\end{array}\right.
$$

respectively. The energy consumption efficiency (EE) and the reporting time-saving efficiency (TE) of the conventional cluster and the proposed CSS schemes compared with the direct CSS scheme can be easily obtained by $\mathrm{EE}_{*}=1-E_{*} / E_{\mathrm{DIR}}$ and $\mathrm{TE}_{*}=1-T_{*} / T_{\mathrm{DIR}}$, respectively, where the asterisk $(*)$ can be replaced by CON or PROP.

When the number of cluster is constant at $n_{c}=5$ as assumed in Figure 5, we can obtain the energy consumption efficiency and the reporting time saving as shown in Figure 6. Obviously, both cluster schemes enable an increase in the energy efficiency and time savings along with the increase in cluster size. As illustrated in Equation 26 and in the simulation result of Figure 6, it can be concluded that the energy efficiency of the proposed scheme is the upper bound of the conventional cluster scheme for all cases of $k$. Therefore, the proposed scheme achieves the highest energy efficiency among cluster schemes.

Similarly, from Figure 7, we can see that the proposed CSS scheme provides higher reporting time savings than the conventional cluster scheme. In fact, for an acceptable value of $l$, i.e., $l=4$, the proposed scheme produces time savings greater than $80 \%$ compared with the conventional direct reporting CSS scheme, while the conventional cluster scheme only remains at $75 \%$ of the highest saving percentage.

\section{Conclusions}

In this paper, we have proposed a cluster-based CSS scheme which includes the selective method in the cluster and the optimal fusion rule in the FC. The proposed selective combination method can dramatically reduce the reporting time and energy consumption while achieving a certain high level of sensing performance espe-

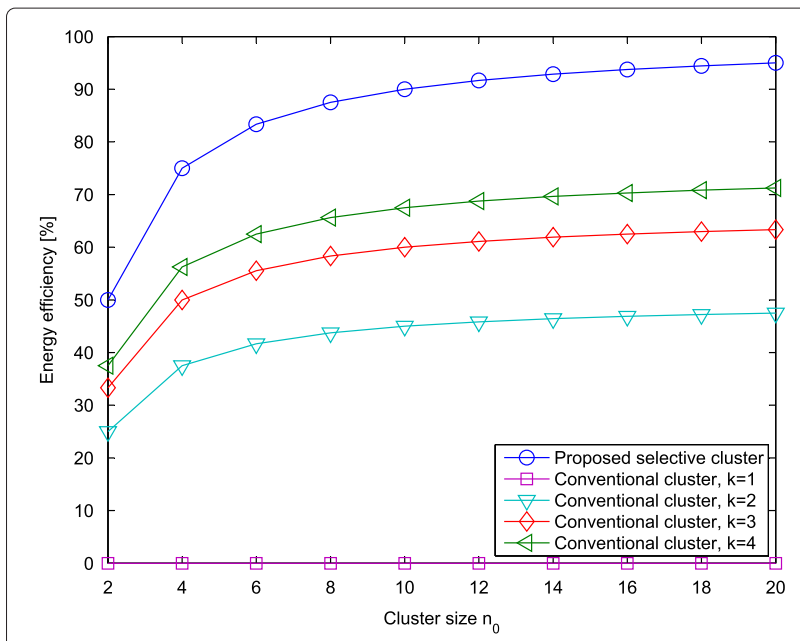

Figure 6 Energy consumption efficiency of the proposed and conventional cluster-based CSS schemes. This efficiency is compared with the conventional direct reporting-based CSS scheme for different cluster sizes and different values of $k$ when the network includes $n_{C}=5$ clusters. 


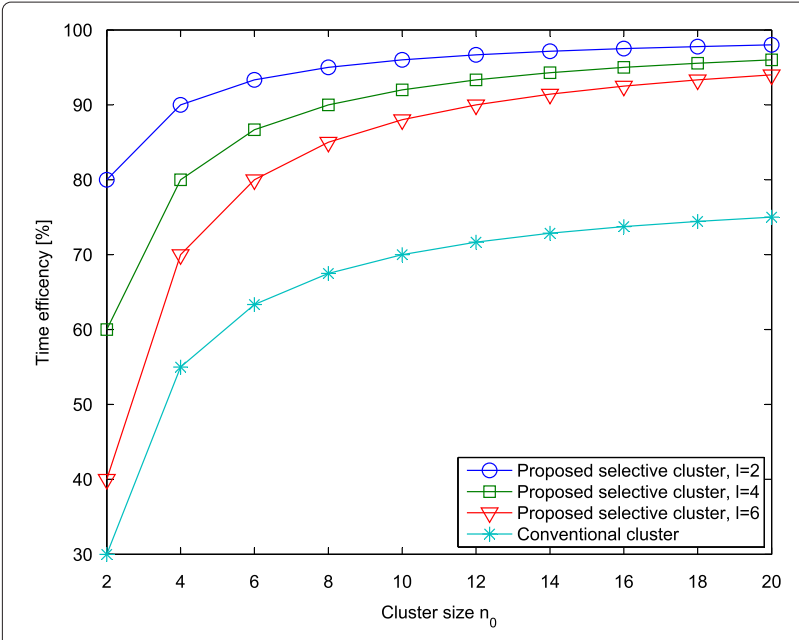

Figure 7 Reporting time saving of the proposed and the conventional cluster-based CSS schemes. This time saving is compared with the conventional direct reporting-based CSS scheme for different cluster sizes and different value of / when the network includes $n_{c}=5$ clusters.

cially when it is combined with the proposed frequency division-based parallel reporting mechanism.

\section{Appendix}

\section{Derivation of Equation 22}

Let $Y$ denote a continuous random variable with PDF $f_{Y}(y)$ and $\operatorname{CDF} F_{Y}(y)$ and let $\left(Y_{1}, Y_{2}, \ldots, Y_{n_{0}}\right)$ be a random sample of size $n_{0}$ drawn from $Y$. The corresponding ordered sample derived from the parent $Y$ is $\left(Y_{(1)}, Y_{(2)}, \ldots, Y_{\left(n_{0}\right)}\right)$, which is arranged in increasing order of absolute value such that $\left|Y_{(1)}\right|<\left|Y_{(2)}\right|<\ldots<$ $\left|Y_{(k)}\right|<\ldots<\left|Y_{\left(n_{0}\right)}\right|$. In order to determine the PDF of $Y_{(k)}$, we define the event $D_{k, y}=\left\{y \leq Y_{(k)} \leq y+\Delta y\right\}=$ $\left\{y \leq \pm\left|Y_{(k)}\right| \leq y+\Delta y\right\}$. Thus, the probability of event $D_{k, y}$ can be calculated by

$$
\begin{aligned}
\operatorname{Pr}\left[D_{k, y}\right] \approx & C_{k}\left(1-F_{Y}(y+\Delta y)+F(-y-\Delta y)\right)^{n_{0}-k} \\
& \times f_{Y}(y) \Delta y\left(F_{Y}(y)-F_{Y}(-y)\right)^{k-1}
\end{aligned}
$$

if $y \geq 0$ or

$$
\begin{aligned}
\operatorname{Pr}\left[D_{k, y}\right] \approx & C_{k}\left(1-F_{Y}(-y)+F_{Y}(y)\right)^{n_{0}-k} f_{Y}(y) \Delta y \\
& \times\left(F_{Y}(-y-\Delta y)-F_{Y}(y+\Delta y)\right)^{k-1}
\end{aligned}
$$

if $y<0$, where $C_{k}=\left(\begin{array}{ccc}n_{0} & \\ k-1 & 1 & n_{0}-k\end{array}\right)$. Consequently, the PDF of $Y_{(k)}$ is calculated using

$$
\begin{aligned}
f_{Y_{(k)}}(y)= & \lim _{\Delta y \rightarrow 0} \frac{\operatorname{Pr}\left[D_{k, y}\right]}{\Delta y}=C_{k}\left(1-\left|F_{Y}(y)-F_{Y}(-y)\right|\right)^{n_{0}-k} \\
& \times\left(\left|F_{Y}(y)-F_{Y}(-y)\right|\right)^{k-1} f_{Y}(y)
\end{aligned}
$$

By replacing $Y$ with $Y^{c_{j}}$ and substituting $k=n_{0}$ into (30), Equation 22 can be obtained.

\section{Competing interests}

Both authors declare that they have no competing interests.

\section{Acknowledgements}

This work was supported by the National Research Foundation of Korea (NRF) grant funded by the Korea government (MEST) (NRF-2012R1A1A2038831 and NRF-2013R1A2A2A05004535).

\section{Author details}

${ }^{1}$ Telecom ParisTech, Paris, France. ${ }^{2}$ University of Ulsan, Ulsan, South Korea.

Received: 16 February 2013 Accepted: 11 June 2013

Published: 24 June 2013

\section{References}

1. D Cabric, SM Mishra, RW Brodersen, Implementation issues in spectrum sensing for cognitive radios. Conf. Record of the 38th Asilomar Conf. on Signals, Systems and Computers. 1, 772-776 (2004)

2. L Chen, J Wang, S Li, in 4th International Symposium on Wireless Communication Systems. An adaptive cooperative spectrum sensing scheme based on the optimal data fusion rule (IEEE Piscataway, 2007), pp. 582-586

3. N Nguyen-Thanh, I Koo, An efficient ordered sequential cooperative spectrum sensing scheme based on evidence theory in cognitive radio. IEICE Trans. Commun. E93.B(12), 3248-3257 (2010)

4. L Hesham, A Sultan, M Nafie, F Digham, Distributed spectrum sensing with sequential ordered transmissions to a cognitive fusion center. IEEE Trans. Signal Process. 60, 2524-2538 (2012)

5. S Maleki, G Leus, Censored truncated sequential spectrum sensing for cognitive radio networks. IEEE J Selected Areas Commun. 31, 364-378 (2013)

6. L De Nardis, D Domenicali, MG Di Benedetto, in 4th International Conference on Cognitive Radio Oriented Wireless Networks and Communications 2009 (CROWNCOM '09). Clustered hybrid energy-aware cooperative spectrum sensing (CHESS) (IEEE Piscataway, 2009), pp. 1-6

7. S Hussain, X Fernando, Approach for cluster-based spectrum sensing over band-limited reporting channels. IET Commun. 6, 1466-1474 (2012)

8. C Guo, T Peng, S Xu, H Wang, W Wang, in IEEE 69th Vehicular Technology Conference 2009 (VTC Spring 2009). Cooperative spectrum sensing with cluster-based architecture in cognitive radio networks (IEEE Piscataway, 2009), pp. 1-5

9. E Karami, AH Banihashemi, in 2011 Ninth Annual Communication Networks and Services Research Conference (CNSR). Cluster size optimization in cooperative spectrum sensing (IEEE Piscataway, 2011), pp. 13-17

10. N Reisi, M Ahmadian, V Jamali, S Salari, Cluster-based cooperative spectrum sensing over correlated log-normal channels with noise uncertainty in cognitive radio networks. IET Commun. 6, 2725-2733 (2012)

11. C Sun, W Zhang, KB Letaief, in Proceedings of IEEE International Conference on Communications, Glasgow. Cluster-based cooperative spectrum sensing for cognitive radio systems (IEEE Piscataway, 2007), pp. 2511-2515

12. N Nguyen-Thanh, I Koo, Log-likelihood ratio optimal quantizer for cooperative spectrum sensing in cognitive radio. IEEE Commun. Lett. 15(3), 317-319 (2011)

13. Z Chair, PK Varshney, Optimal data fusion in multiple sensor detection systems. IEEE Trans. Aerospace and Electronic Syst. AES-22(1), 98-101 (1986)

14. B Vo Nguyen Quoc, TQ Duong, D Benevides da Costa, GC Alexandropoulos, A Nallanathan, Cognitive amplify-and-forward relaying with best relay selection in non-identical Rayleigh fading. IEEE COML. 17(3), 475-478 (2013) 
15. TQ Duong, VNQ Bao, H Tran, GC Alexandropoulos, HJ Zepernick, Effect of primary networks on the performance of spectrum sharing AF relaying. IET Lett. 48(1), 25-27 (2012)

16. H Urkowitz, Energy detection of unknown deterministic signals. Proceedings of the IEEE. 55(4), 523-531 (1967)

17. M Barkat, Signal Detection and Estimation, 2nd edn. (Artech House, London, 2005)

18. SJ Shellhammer, SN Shankar, R Tandra, J Tomcik, in Proceedings of the 1st International Workshop on Technology and Policy For Accessing Spectrum, vol. 222. Performance of power detector sensors of DTV signals in IEEE 802.22 WRANs (ACM Press New York, 2006)

\section{doi:10.1186/1687-1499-2013-176}

Cite this article as: Nguyen-Thanh and Koo: A cluster-based selective cooperative spectrum sensing scheme in cognitive radio. EURASIP Journal on Wireless Communications and Networking 2013 2013:176.

\section{Submit your manuscript to a SpringerOpen ${ }^{\circ}$ journal and benefit from: \\ - Convenient online submission \\ - Rigorous peer review \\ - Immediate publication on acceptance \\ - Open access: articles freely available online \\ - High visibility within the field \\ - Retaining the copyright to your article \\ Submit your next manuscript at $\boldsymbol{\triangleright}$ springeropen.com}

